\title{
Axial Organization of a Brain Region That Sequences a Learned Pattern of Behavior
}

\author{
Tiffanie R. Stauffer, Kevin C. Elliott, Matthew T. Ross, Mark J. Basista, Richard L. Hyson, and Frank Johnson \\ Program in Neuroscience and Department of Psychology, Florida State University, Tallahassee, Florida 32306-4301
}

Neural activity within HVC (proper name), a premotor nucleus of the songbird telencephalon analogous to premotor cortical regions in mammals, controls the temporal structure of learned song in male zebra finches (Taeniopygia guttata). HVC is composed of a superficially isomorphic neuronal mosaic, implying that song is encoded in a distributed network within HVC. Here, we combined HVC microlesions ( $10 \%$ focal ablation) with singing-driven immediate-early gene (IEG) labeling to explore the network architecture of HVC during singing. Microlesions produce a transient disruption of HVC activity that results in a temporary ( $\sim 1$ week) loss of vocal patterning. Results showed an asymmetrical reduction in the density of IEG-labeled cells 3-5 d after microlesions: swaths of unlabeled cells extended rostrally and/or caudally depending on the position of the HVC microlesion. Labeling returned once birds recovered their songs. Axial swaths of unlabeled cells occurred whether microlesions were located at rostral or caudal poles of HVC, indicating that the localized reduction in IEG labeling could not be attributable solely to transection of afferents that enter HVC rostrally. The asymmetrical pattern of reduced IEG labeling could be explained if synaptic connectivity within HVC is organized preferentially within the rostrocaudal axis. In vivo retrograde tracer injections and in vitro stimulation and recording experiments in horizontal slices of HVC confirmed a rostrocaudal organization of HVC neural connectivity. Our findings suggest that HVC contains an axially organized network architecture that may encode the temporal structure of song.

\section{Introduction}

Zebra finch song is a learned sequence of sounds produced by dual premotor pathways that traverse the posterior and anterior telencephalon. The posterior pathway contains regions analogous to mammalian premotor cortex [HVC (a proper name)] and primary motor cortex [robust nucleus of the arcopallium (RA)]. HVC generates the time-locked neural activity that drives the learned sequence of sounds (Hahnloser et al., 2002; Long and Fee, 2008). The anterior pathway includes a basal ganglia circuit that generates time-variant neural activity and drives variable sequences of sound (Aronov et al., 2008), contributing both degenerative and adaptive variability to song (Brainard and Doupe, 2000; Andalman and Fee, 2009). Presynaptic terminals from both pathways converge on the dendrites of RA neurons, which integrate activity conveyed by the dual premotor pathways (Mooney and Konishi, 1991).

HVC lies within the dorsal surface of the caudal nidopallium (Fig. 1) and is composed of three hodologically defined subpopulations (RA-projecting, Area X-projecting interneurons) ar-

\footnotetext{
Received Feb. 28, 2012; revised April 27, 2012; accepted May 10, 2012.

Author contributions: T.R.S., R.L.H., and F.J. designed research; T.R.S., K.C.E., M.T.R., M.J.B., R.L.H., and F.J. performed research; T.R.S., R.L.H., and F.J. analyzed data; T.R.S., R.L.H., and F.J. wrote the paper.

This work was supported by National Institutes of Health Grant DC02035. We thank Dr. Richard Bertram and Dr. Wei Wu for assistance in the design of the experiments, Ross Henderson and Paul Hendrick for the design and fabrication of the songbird recording chambers, and Dr. Ofer Tchernichovski for use of the SA+ software. We also thank three anonymous reviewers for their important contributions to the organization and content of this manuscript.

Correspondence should be addressed to Frank Johnson, Program in Neuroscience and Department of Psychology, Florida State University, Tallahassee, Florida 32306-4301. E-mail: johnson@psy.fsu.edu.

DOI:10.1523/JNEUROSCI.0978-12.2012

Copyright $\odot 2012$ the authors $\quad 0270-6474 / 12 / 329312-11 \$ 15.00 / 0$
}

ranged in a superficially isomorphic mosaic that includes regional differences in cytoarchitecture (Fortune and Margoliash, 1995; Wild et al., 2005). Superficially isomorphic anatomy and a similar neural response to autogenous song across the spatial extent of HVC suggest that song is encoded in a uniform distributed manner within HVC (Margoliash, 1986; Sutter and Margoliash, 1994). However, recent computational models based on single-unit recordings from HVC of singing birds suggest encoding within chain architectures, which could introduce spatial asymmetry in encoding (Long et al., 2010). In the present study, we explored the network architecture of HVC in singing birds by combining HVC microlesions ( $10 \%$ focal ablation) with immediately-early gene (IEG) labeling of HVC neurons. Microlesions produce a transient disruption of HVC network activity and result in a temporary ( $\sim 1$ week) loss of vocal patterning (Thompson and Johnson, 2007; Thompson et al., 2007). If song is encoded by a uniform distributed network, we hypothesized that microlesions would produce a symmetrical reduction in IEG labeling throughout HVC.

Experimental findings revealed a $>50 \%$ reduction in the density of singing-driven IEG labeling 3-5 d after HVC microlesions that returned to control levels in birds allowed to recover their vocal pattern. However, the observed reduction in IEG labeling was asymmetrical and attributable in part to a distinctive swath of reduced IEG labeling rostral and/or caudal to the position of the microlesion. These axial swaths of unlabeled cells were observed whether microlesions were located at rostral or caudal poles of HVC, indicating that the patterned reduction in IEG labeling could not be explained solely by transection of afferent fibers that enter HVC rostrally. The asymmetrical pattern of reduced IEG 


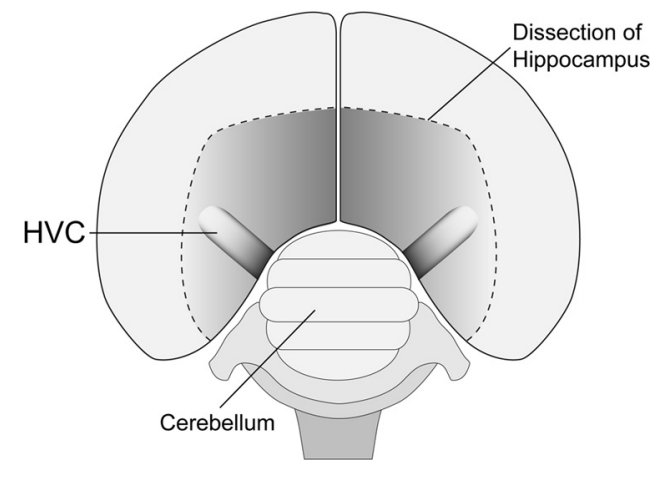

Figure 1. Dorsal perspective schematic of an adult male zebra finch brain shows the position of HVC within the dorsal surface of the caudal nidopallium. Approximate axial dimensions of HVC are $\sim 1.5 \mathrm{~mm}$ (mediolateral) $\times \sim 900 \mu \mathrm{m}$ (rostrocaudal) $\times \sim 500 \mu \mathrm{m}$ (dorsoventral). In coronal or sagittal sections, HVC is ovoid in shape, with cell populations organized in a superficially isomorphic mosaic. For additional details, see Materials and Methods and Fortune and Margoliash (1995).

labeling could be explained if physiological activity within HVC is organized preferentially within the rostrocaudal axis. In vivo retrograde tracer injections (which extended the previous findings of Nottebohm et al., 1982) and in vitro stimulation and recording experiments in horizontal slices of HVC confirmed preferential projections and physiological activity within the rostrocaudal axis. Our findings do not support a uniform distributed model of encoding and instead provide the first functional evidence of axially organized network architecture within HVC.

\section{Materials and Methods}

\section{Animals and environment}

Adult male zebra finches $(n=31,>90 \mathrm{~d}$ after hatch) were raised in our breeding colony and individually caged in visual but not auditory isolation from each other. The cages were placed in acoustic chambers equipped with recording microphones that captured vocal behavior throughout the experiment. All birds were provided access to food and water ad libitum throughout the experiment and housed on a $14 / 10$ light/dark photoperiod. Birds were allowed 1 week to acclimate to the acoustic chambers before song recording ensued. All daily care and experimental procedures of the birds were reviewed and approved by the Florida State University Animal Care and Use Committee.

\section{HVC microlesion surgery}

After the 1 week acclimation period and a minimum of three preoperative recording days, $n=13$ birds received bilateral HVC microlesions. Birds were deeply anesthetized with Equithesin $(0.05 \mathrm{cc})$ and then secured in a stereotaxic instrument. The skull was exposed by making an incision down the center of the scalp and retracting the skin using curved forceps. The bifurcation at the midsagittal sinus was used as stereotaxic zero, and a small craniotomy over left and right HVC was made using predetermined coordinates (Thompson and Johnson, 2007). Electrolytic lesions were administered using two electrode holders placed $4 \mathrm{~mm}$ apart that secured two Teflon-insulated tungsten electrodes, enabling two simultaneous penetrations each $2 \mathrm{~mm}$ lateral from the midline. Each penetration site had a depth of $0.6 \mathrm{~mm}$, and a $100 \mu \mathrm{A}$ current was passed through each electrode for $60 \mathrm{~s}$. Previous studies using a similar technique have shown that this produces a $\sim 10 \%$ ablation by volume of each HVC (Thompson and Johnson, 2007; Thompson et al., 2007). Once the lesions were completed, the incision was closed using veterinary adhesive and birds were returned to their home cages.

\section{IEG immunocytochemistry}

HVC microlesion and control (CTL) birds were overdosed with Equithesin $(0.08 \mathrm{cc})$ and perfused transcardially with saline $(20 \mathrm{ml})$, followed by ice-cold $4 \%$ paraformaldehyde $(40 \mathrm{ml})$. Dissected brains were postfixed overnight in $4 \%$ paraformaldehyde, followed by PBS until sectioning.

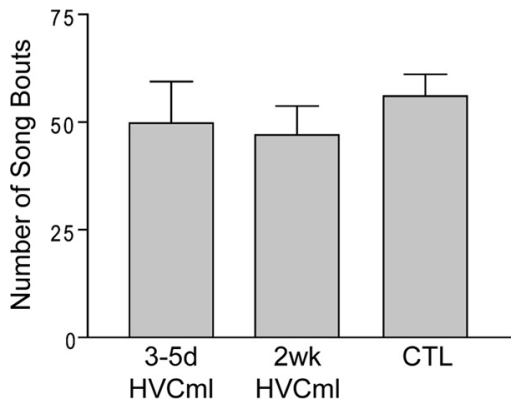

Figure 2. All birds processed for singing-driven ZENK (IEG) labeling produced a similar number of song bouts before perfusion. For group name definitions, see Materials and Methods. Columns are means $\pm \mathrm{SE}$.

Brains were sectioned serially in coronal or sagittal planes using a vibratome (Leica VT1000S) at a section thickness of $40 \mu \mathrm{m}$. Immunoreactivity for the IEG ZENK (known as EGR-1 in mammals) was examined at the following time points after microlesion: $3-5 \mathrm{~d}(3-5 \mathrm{~d} \mathrm{HVCml} ; n=9)$ or 2 weeks ( $2 \mathrm{wk}$ HVCml; $n=4)$. Time points were chosen to capture birds in different phases of vocal recovery, such that 3-5d HVCml birds were still in the process of vocal recovery and $2 \mathrm{wk} \mathrm{HVCml}$ birds had recovered their vocal pattern. CTL birds $(n=5)$ did not receive surgery and were assessed after a 1 week acclimation period and $3 \mathrm{~d}$ of behavioral recording. The amount of singing on the day of perfusion was matched for all groups ( $\sim 50$ songs during the first hour of morning singing; Fig. 2 ) because the amount of singing is highly correlated with IEG expression levels (Jarvis et al., 1998).

For both right and left HVC of each bird, every fourth tissue section that contained HVC was selected and processed for detection of ZENK protein. This resulted in 6-10 sections per HVC, depending on coronal versus sagittal planes of section. Immunocytochemistry was conducted using procedures published previously (Whitney et al., 2000; Whitney and Johnson, 2005). Briefly, free-floating sections were pretreated in $1 \%$ hydrogen peroxide for $30 \mathrm{~min}$, rinsed in PBS, and then pretreated with $5 \%$ normal goat serum (NGS) (Vector Laboratories) for $30 \mathrm{~min}$. Sections were rinsed in PBS solution and then incubated in EGR-1 (ZENK) antibody serum overnight (Santa Cruz Biotechnology; 1:1000 dilution: $10 \mu \mathrm{l}$ of EGR-1 antibody, $10 \mathrm{ml}$ of $0.3 \%$ Triton X-100 solution, and $100 \mu \mathrm{l}$ of $5 \%$ NGS). This antibody is a rabbit polyclonal, raised against a peptide derived from the last 19 aa residues of the $\mathrm{C}$ terminus of rodent and human EGR-1, which shows a high degree of conservation in songbirds. Moreover, specific labeling against zebra finch ZENK has been demonstrated by Western blot (Mello and Ribeiro, 1998). Negative control sections, in which processing included all components save the primary antibody, showed no evidence of the nuclear labeling that is characteristic of ZENK.

The following day, sections were rinsed in PBS solution and incubated in secondary antibody biotinylated anti-rabbit IgG serum (Vector Laboratories; 1:200 dilution: $50 \mu \mathrm{l}$ of biotinylated anti-rabbit IgG, $10 \mathrm{ml}$ of $0.3 \%$ Triton X-100 solution, and $150 \mu$ l of 5\% NGS) for $1 \mathrm{~h}$. Sections were rinsed and treated with avidin-biotin complex reagent (Vector Laboratories) for $1 \mathrm{~h}$, rinsed again, and reacted in $\mathrm{DAB}+\mathrm{H}_{2} \mathrm{O}_{2}$ serum (Vector Laboratories). Sections were rinsed a final time, mounted onto slides with gelatin mounting solution, and coverslipped the next day.

\section{Microlesion verification}

Comparison with thionin-stained alternate sections and thionin counterstaining of IEG-labeled sections conducted after completion of cell density measurements indicated that the IEG-defined borders of HVC aligned with the Nissl-defined borders of HVC as described by Fortune and Margoliash (1995). Serial sections processed for IEG immunoreactivity were examined using a digital camera attached to a light microscope (Leica DM5000B) at low power $(5 \times)$ and each bird that received HVC microlesions had the remaining volume of HVC and the volume of the microlesion estimated using Neurolucida software. This was done for HVC in both the left and right hemispheres using procedures reported 
previously (Thompson and Johnson, 2007). Briefly, the total remaining bilateral area of HVC was estimated by tracing the immunodefined borders of HVC in each section that it appeared. The microlesion area within HVC was traced separately. In many cases, the spatial extent of microlesions obscured visualization of portions of the HVC border, particularly the ventral border and the shelf region that surrounds the ventral border of HVC (Fortune and Margoliash, 1995). These portions of the HVC border were inferred based on the characteristic ovoid shape of HVC in coronal or sagittal sections, as well as comparison with HVC borders in adjacent sections that did not contain microlesion damage. Although the HVC shelf receives input from Field L (the avian analog of primary auditory cortex in mammals) and may play a role in the conveyance of auditory information to HVC (Fortune and Margoliash, 1995), microlesion damage that extends into this region does not prevent vocal recovery (Thompson and Johnson, 2007; Thompson et al., 2007; present results).

To calculate the percentage remaining volume of HVC, the following equation was used: remaining volume of $\mathrm{HVC} /$ (remaining volume of $\mathrm{HVC}+$ microlesion volume). During this analysis, we also verified that the locations of the microlesions within HVC were bilaterally symmetrical within birds, although we noted that the rostrocaudal position of the microlesions within HVC varied across birds. However, consistent with previous observations (Thompson and Johnson, 2007; Thompson et al., 2007), the rostrocaudal position of the HVC microlesions was not related to specific behavioral outcomes.

\section{Cell density measurements}

Visual examination of the effect of microlesions on IEG labeling in HVC of the $3-5 \mathrm{~d} H V C m l$ birds revealed that regions within the rostrocaudal axis of the microlesion showed a far greater reduction in IEG labeling than regions lying medial or lateral to the lesion site. That is, there was substantial variation in the density of IEG labeling within HVC of 3-5d HVCml birds, which we quantified by creating 2-D binary images of IEG labeling in sections that contained HVC (Fig. 3). Although quantification with 2-D binary images allows complete capture of spatial variation in the density of IEG labeling, it does effectively collapse the section thickness (in our case, $40 \mu \mathrm{m}$ ), resulting in a likely underestimate of the total number of labeled cells in each HVC. However, because we used this approach in all birds in all groups, large relative differences in the density of IEG labeling between groups will be preserved.

To generate 2-D binary images of HVC, every IEG-labeled section that contained HVC was imaged using an Optronics MicroFIRE digital camera connected to a Leica DM 5000B microscope equipped with a motorized stage drive, coupled to a personal computer running Stereo Investigator 6.0 and Neurolucida software (MicroBrightField). To ensure that individual IEG-labeled cells could be properly resolved, multiple images were taken using a $20 \times$ objective throughout each HVC and then merged to create a high-resolution photo montage of each HVC. Given that the montage included some of the area surrounding HVC, images were loaded into Adobe Photoshop CS2 (Adobe Systems) and cropped to only include HVC. The section was then converted into a binary image by using the threshold function in Photoshop (Fig. 3). Because thresholding is a manual step, two individual observers (blind to the groups) independently determined the appropriate threshold level for each section. All binary images were then loaded into Image Pro Plus software (MediaCybernetics), and cells were counted using the automated cell-counting function. Next, the volume of each HVC section (which included the microlesion, when present) was determined by tracing the cropped image sections in Neurolucida to obtain the area for each section and then multiplying that measurement by section thickness. The labeling density in HVC sections was then rank-ordered for
Bird 96 (3-5d HVCml)
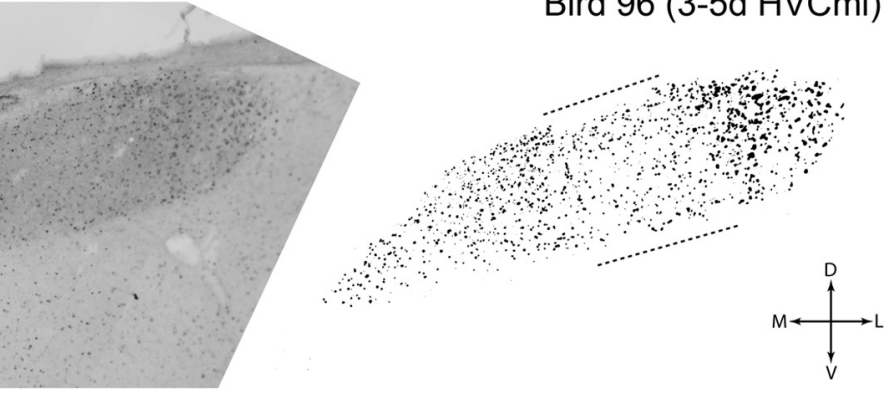

Figure 3. High-resolution bright-field composite images of HVC were used to generate binary images of singing-driven ZENK labeling for quantification. Shown is an image of singing-driven IEG labeling in a coronal section from the rostral half of HVC, taken from a bird that was processed $3 \mathrm{~d}$ after HVC microlesions ( $3-5 \mathrm{~d} \mathrm{HVCml} \mathrm{group).} \mathrm{The} \mathrm{bright-field} \mathrm{image} \mathrm{at} \mathrm{left} \mathrm{is} \mathrm{a} \mathrm{composite}$ f multiple bright-field images captured with a $20 \times$ objective. At right is the binary image of IEG labeling that was extracted from

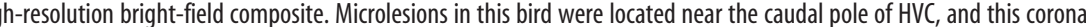
section (which is $480 \mu \mathrm{m}$ rostral of the microlesion) shows a region of reduced IEG labeling (bracketed by dashed lines) that aligned with the mediolateral position of the microlesion. For additional details on quantification of IEG labeling, see Materials and medial; V, ventral.

each bird (i.e., ordering sections with the highest density to the lowest density) to check for inter-observer reliability. No differences were found between the two observers, and results were calculated for each bird using an average of the HVC density measures that were generated by the two observers. An overall density measure (IEG-labeled cells per cubic millimeter) for each HVC was obtained by the following formula: total number of IEG-labeled cells/total volume of IEG-labeled sections.

\section{Spatial distribution of IEG labeling}

Using Neurolucida software and the same microscope and camera described above, we mapped the spatial distribution of IEG-labeled neurons in HVC of $n=4$ birds. Birds were selected based on rostral versus caudal positions of the HVC microlesion and coronal versus sagittal planes of section ( $3-5 \mathrm{~d}$ HVCml birds, $n=2$; CTL birds, $n=2$ ). The reconstructions also included the location of the HVC microlesion in the 3-5d HVCml birds.

In each IEG-labeled section that contained HVC, the contour function in Neurolucida was used to trace the borders of HVC with a low-power $(5 \times)$ objective. With the aid of a higher-power objective $(20 \times)$, a cell marker was used to label the position of all IEG-labeled nuclei present in each section. An observer without knowledge of the experimental manipulation completed this task in the HVCml birds. Serial sections of HVC were then aligned to one another by using separate contours to trace the outer surface of the surrounding caudal telencephalon and RA (when present). RA appeared in many of the same sections as HVC and is spherical in shape, providing a known point of reference. RA and the outer surface of the caudal telencephalon were used to align contours during the reconstruction of HVC. As a final step, reconstructions were rotated and viewed from a dorsal perspective to verify proper alignment relative to the midline (Fig. 1). In HVCml birds, these reconstructions contained the spatial pattern of IEG labeling relative to the position of the HVC microlesion.

Following the method of Olson et al. (2011), tools in Neurolucida were used to quantify the density of IEG labeling across the mediolateral axis of each coronal section (or the rostrocaudal axis of each sagittal section) at $100 \mu \mathrm{m}$ increments. For each bird's HVC, these values were then normalized to the $100 \mu \mathrm{m}$ segment with the highest density of IEG labeling. This procedure was used to quantify spatial differences in the density of IEG labeling across the mediolateral and rostrocaudal extent of HVC.

\section{Song analysis}

In this study, all songs were "undirected" (i.e., not directed toward a female), because male birds were caged individually throughout the experiment. Behavioral measures were taken at three stages: preoperative (PRE1, PRE2), the first day of postoperative singing (POST1), and the final day of postoperative singing (FINAL).

Song recording. Recording chambers were outfitted with either overhead microphones or unidirectional microphones placed at the front end 
of each cage. The vocalizations of all birds were recorded continuously using a personal computer running sound-event triggered software (Avisoft Recorder; Avisoft Bioacoustics) that sampled sound at a rate of 44 $\mathrm{kHz}$. Each captured sound event was saved to the hard drive of the computer as a time-stamped .wav file, and each day of singing by each bird was saved as a single directory that was then backed up onto a second hard drive. To ensure that all song bouts were recorded each day, capture settings on Avisoft Recorder was set to favor false positives (e.g., a series of calls or repeated pecking on the food dish). To eliminate files that did not contain song bouts, we followed methods described by Wu et al. (2008). Briefly, all .wav files were converted to .jpg images of the frequency spectra present in each file using the batch function in Spectrogram 12.0 (Visualization Software, Richard Horne, Stafford, VA). The .jpg images of the frequency spectrograms were then sorted using an image-pattern matching program (iMatch; Mario Westphal, Usingen, Hessen, Germany) to isolate and group .wav files that did not contain singing. All .wav files that did not contain singing were then batch deleted using MATLAB script (MathWorks).

Perfusion day recording. Songs were carefully tracked on perfusion day by an observer monitoring the personal computer running sound-eventtriggered software. Birds were allowed to sing for 1 hour after lights on, after which they were removed from their cages and overdosed with Equithesin $(0.08 \mathrm{cc})$. The amount of singing produced during this $1 \mathrm{~h}$ period was similar for all groups (Fig. 2).

Similarity scores. Sound Analysis Pro $(\mathrm{SA}+$ ) 1.04 software (Tchernichovski et al., 2000) was used to assess change in the acoustic structure of songs, using the default zebra finch settings. The similarity score function in SA+ was used to compare singing produced at PRE1 to other pre- and post-operative days of singing. For each bird, we selected a representative canonical motif from PRE1 singing and compared it to the first 50 song bouts captured at PRE1, PRE2, POST1, and FINAL. To quantify the recovery of acoustic structure, the average PRE1 similarity score was used as a baseline, and the average similarity scores from the subsequent days (PRE2, POST1, and FINAL) were each divided by the average PRE1 similarity score to calculate a percentage similarity to PRE1.

\section{Statistical analysis of anatomy and song}

Anatomical analysis. Comparison of HVC microlesion size was performed for 3-5d $\mathrm{HVCml}$ and $2 \mathrm{wk} \mathrm{HVCml}$ birds using a one-way ANOVA (group $\times$ percentage bilateral HVC damage). One-way repeated-measures ANOVAs (left damage $\times$ right damage) were conducted within each HVC microlesion group to analyze differences in lesion size across the two HVCs. Quantification of IEG labeling for all HVC microlesion groups was measured as a percentage of the mean IEG labeling in CTL birds. A one-way ANOVA (group $\times$ percentage CTL labeling) was conducted to analyze group differences in the density of IEG-labeled cells in HVC.

Song analysis. Similarity scores for singing at PRE2, POST1, and FINAL were expressed as a percentage of PRE1. A separate one-way repeated-measure ANOVA $($ PRE $1 \times$ PRE $2 \times$ PRE $1 \times$ FINAL) was conducted for each group to analyze changes in song phonology. All pairwise comparisons of statistically significant $(p<0.05)$ main effects or interactions were evaluated using Student-Newman-Keuls tests. All statistical ANOVA analyses were performed using SigmaStat 2.03 (SPSS).

\section{In vivo injections of retrograde tracers into HVC}

Using stereotaxic surgical techniques, focal pressure injections of retrograde tracers $(\sim 200 \mathrm{nl})$ were made into HVC of adult male zebra finches $(\mathrm{n}=8)$. Birds were deeply anesthetized with Equithesin $(0.05 \mathrm{cc})$ and secured in the stereotaxic instrument, and the skull was exposed by making an incision down the center of the scalp and retracting the skin using curved forceps. The bifurcation at the midsagittal sinus was used as stereotaxic zero, and a small craniotomy over left and right HVC was made using predetermined coordinates. A visually guided approach was then used to optimize targeting of focal tracer injections into HVC. That is, with the aid of a surgical microscope, the hippocampus overlying HVC on one side of the brain (left or right) was carefully resected and folded medially to visualize the position of HVC on one side of the brain (Fig. 1).
Table 1. HVC microlesion morphology

\begin{tabular}{lllllll}
\hline & & \multicolumn{2}{l}{ \% Damage } & & \multicolumn{2}{l}{ \% Remaining } \\
& \% Bilateral damage & Left & Right & & Left & Right \\
\hline $3-5 d$ & $13 \pm 2$ & $15 \pm 4$ & $10 \pm 2$ & & $85 \pm 4$ & $90 \pm 2$ \\
2 weeks & $10 \pm 2$ & $14 \pm 5$ & $11 \pm 5$ & & $86 \pm 5$ & $89 \pm 5$ \\
\hline
\end{tabular}

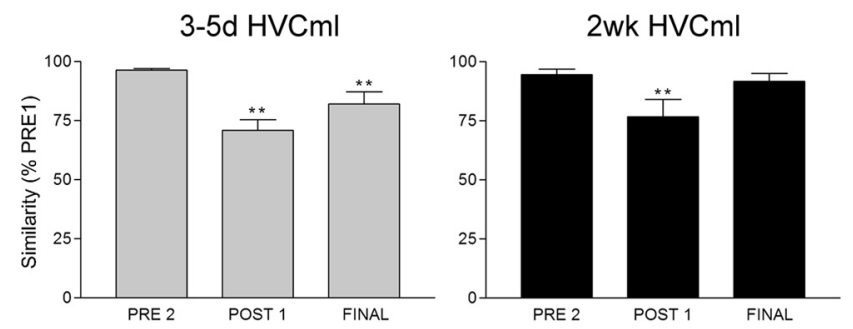

Figure 4. Similarity scores for the two groups of birds that received HVC microlesions show the time course of vocal recovery. When similarity scores at PRE2, POST1, and FINAL were expressed as a percentage of the similarity score measured at PRE1, within-group repeatedmeasures ANOVAs were highly significant for each group ( $F>10, p<0.01$, all groups). All groups showed stable singing at PRE2, and all groups were destabilized on POST1 ( ${ }^{* *} p<0.01$ for all groups). However, singing produced by birds in the $3-5 \mathrm{~d} \mathrm{HVCml} \mathrm{group} \mathrm{on} \mathrm{the} \mathrm{day} \mathrm{of}$ perfusion (FINAL) remained significantly different from PRE1 singing $\left({ }^{* *} p<0.01\right)$, meaning that 3-5 d provided an insufficient amount of time for birds in this group to fully recover their preoperative song pattern. In contrast, birds in the $2 \mathrm{wk} \mathrm{HVCml} \mathrm{group} \mathrm{showed} \mathrm{a} \mathrm{full} \mathrm{recovery} \mathrm{of}$ their vocal patterns, because similarity scores on the day of perfusion (FINAL) were not significantly different from PRE1 $(p=0.30)$. These data replicate previous findings by Thompson and Johnson (2007) and Thompson et al. (2007). Columns are means \pm SE.

Because HVC anatomy is bilaterally symmetrical, stereotaxic coordinates obtained from the exposed HVC were used to target tracer injections into HVC on the opposite side of the brain (in which the hippocampus remained intact). Tracers were delivered into HVC via a glass micropipette attached to a gas pressure injection system (Applied Scientific Instrumentation MPPI-3) at a depth of $0.4 \mathrm{~mm}$ from the surface of the brain. Tracer injections were made exclusively into the HVC in which the hippocampus remained intact as the hippocampal resection procedure could potentially alter the dynamics of dye transport in the underlying HVC. After tracer injection, the resected hippocampus was folded back toward the midline, and the incision was closed using veterinary adhesive.

Several different retrograde tracers were used to ensure that the observed pattern of HVC labeling was not tracer specific (Fluorogold, Fluorochrome; fast blue, Polysciences; DiO, Life Technologies). In all cases, the volume of tracer was small $(\sim 10 \mathrm{nl})$ and ejected in a single step to obtain focal placement of dyes. In particular, use of the hydrophobic tracer $\mathrm{DiO}$ resulted in highly focal injection sites. Retrograde tracers were used so that the borders of injection sites could be clearly observed. Small tracer volumes and the focal injection sites produced by single-step injections were critical to mapping retrograde labeling within HVC.

After a 4-6 d period for tracer transport, birds were overdosed with Equithesin $(0.08 \mathrm{cc})$ and perfused transcardially with saline $(20 \mathrm{ml})$, followed by ice-cold $4 \%$ paraformaldehyde $(40 \mathrm{ml})$. Dissected brains were postfixed overnight in $4 \%$ paraformaldehyde, followed by PBS until sectioning. Brains were sectioned serially in the coronal plane using a vibratome (Leica VT1000S), and tissue sections containing HVC were mounted on glass slides and imaged for fluorescent labeling using a digital camera attached to a Leica DM5000B microscope. Serial sections containing HVC were imaged either at low ( $5 \times$ objective) or high $(10 \times$ objective) magnification on an alternating basis. After fluorescent imaging, sections were stained with thionin and imaged again under brightfield illumination to visualize the Nissl-defined borders of HVC.

In vitro electrophysiology in HVC slices

Preparation of brain slices. Horizontal slices were obtained from adult male zebra finches $(n=5)$ to allow comparisons of electrophysiological connectivity in both rostrocaudal and mediolateral axes. Birds were 
deeply anesthetized in a bell jar with isoflurane and killed by rapid decapitation. The brain was extracted from the skull and transferred to a dissection dish containing ice-cold artificial CSF (ACSF). ACSF contained the following (in mM): $130 \mathrm{NaCl}, 26 \mathrm{NaH} 2 \mathrm{CO}_{3}, 3 \mathrm{KCl}, 3 \mathrm{CaCl}_{2}, 1$ $\mathrm{MgCl}_{2}, 1.25 \mathrm{NaH}_{2} \mathrm{PO}_{4}$, and 10 dextrose (constantly gassed with $95 \%$ $\mathrm{O}_{2} / 5 \% \mathrm{CO}_{2}$ ), $\mathrm{pH}$ 7.4. The brain was bisected along midline, and the hippocampus overlying $\mathrm{HVC}$ was peeled away from the brain using fine forceps. The hemisphere was then mounted to a custom-made tilting stage of a vibrating blade tissue slicer using cyanoacrylate glue. The brain was positioned in a parahorizontal plane such that the plane of the blade formed a tangent with the peak of the protrusion formed by $\mathrm{HVC}$ on the surface of the brain. A 300- to $400-\mu \mathrm{m}$-thick slice was taken, transferred to a submersion-type recording chamber, and perfused $(2-3 \mathrm{ml} / \mathrm{min}$ by gravity) with room temperature oxygenated ACSF.

Recording. Horizontal slices of HVC were visualized using an upright fixed-stage microscope with a $10 \times$ objective and $10 \times$ eyepieces fit with an eyepiece reticule. HVC is heavily myelinated compared with the surrounding nidopallium, which provided sufficient contrast to unambiguously discern the borders of HVC in the slice. A bipolar stimulating electrode, constructed from a pair of Tefloncoated platinum wires, was placed on HVC in a position that would allow the recording electrode to be placed in either the rostrocaudal or mediolateral axis. Stimulation was provided by a constant-current stimulator controlled through the Clampex 9.2 software (Molecular Devices). Each stimulus was a $20 \mu \mathrm{s}$ monophasic current pulse. Intensity was held constant at $0.3 \mathrm{~mA}$ for all comparisons of the driven responses. This amplitude was selected based on preliminary studies showing its ability to drive robust responses in distal regions of HVC without masking responses with stimulation artifact. Stimulation pulses were presented in trains of 20 pulses at $5 \mathrm{~Hz}$.

Electrically driven field potential recordings were obtained using glass micropipettes (3-7 $\mathrm{M} \Omega$ ) filled with ACSF. The amplified (either $10 \times$ DC or $100 \times$ AC, Multiclamp 700B; Molecular Devices) signals were digitized at 100 $\mathrm{kHz}$, low-pass filtered at $10 \mathrm{kHz}$, and stored for subsequent offline analysis (ClampFit 9.2, Molecular Devices). Recordings made in DC mode were additionally high-pass filtered at $0.1 \mathrm{~Hz}$ to eliminate artifact from slow DC shifts. The same recording electrode was placed sequentially in different positions in HVC while holding the stimulating position and stimulation intensity constant. Recordings at locations equidistant from the stimulating electrode were analyzed and compared for statistical analyses. The peak-to-peak amplitudes of averaged field potentials ( $n=20$ traces per average) for recordings in the rostrocaudal and mediolateral axes were compared using a paired $t$ test. Both presynaptic and postsynaptic potentials were analyzed separately. The division between presynaptic and postsynaptic responses was placed at the time of the maxima after the first negativity that occurred within $1.5 \mathrm{~ms}$ after the stimulation onset. In recordings with no obvious presynaptic response, the division was placed at the time used for recordings from that slice at locations that did have a presynaptic potential. Replacing the circulating medium with ACSF containing low $\mathrm{Ca}^{2+}(0.5 \mathrm{~mm})$ and high $\mathrm{Mg}^{2+}(5 \mathrm{~mm})$ concentrations, eliminated field potentials that occurred more than $\sim 1.5 \mathrm{~ms}$ after the onset of the stimulation artifact (data not shown), confirming that later responses were not driven directly by the electrical stimulation. Different slices were placed in the chamber at different orientations, so the pattern of results is not attributable to orientation of the slice relative to fluid inflow or relative to the orientation of the poles of the bipolar electrode. In one case, the slice was rotated after the first series of record-

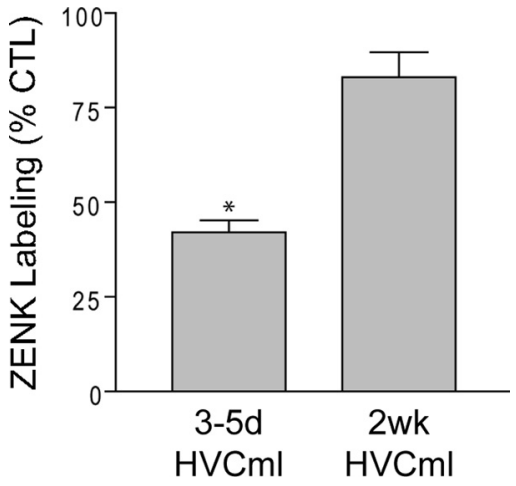

Figure 5. Singing-driven ZENK (IEG) labeling in HVC was significantly reduced 3-5 d after HVC microlesions. Measured as a percentage of labeling in CTL birds, the density of IEG labeling in 3-5d HVCml birds was significantly reduced relative to all other groups (CTL, 2 wk HVCml, ${ }^{*} p<0.05$ all comparisons). 2 wk HVCml birds were not significantly different from (TL birds. Columns are means $\pm \mathrm{SE}$.
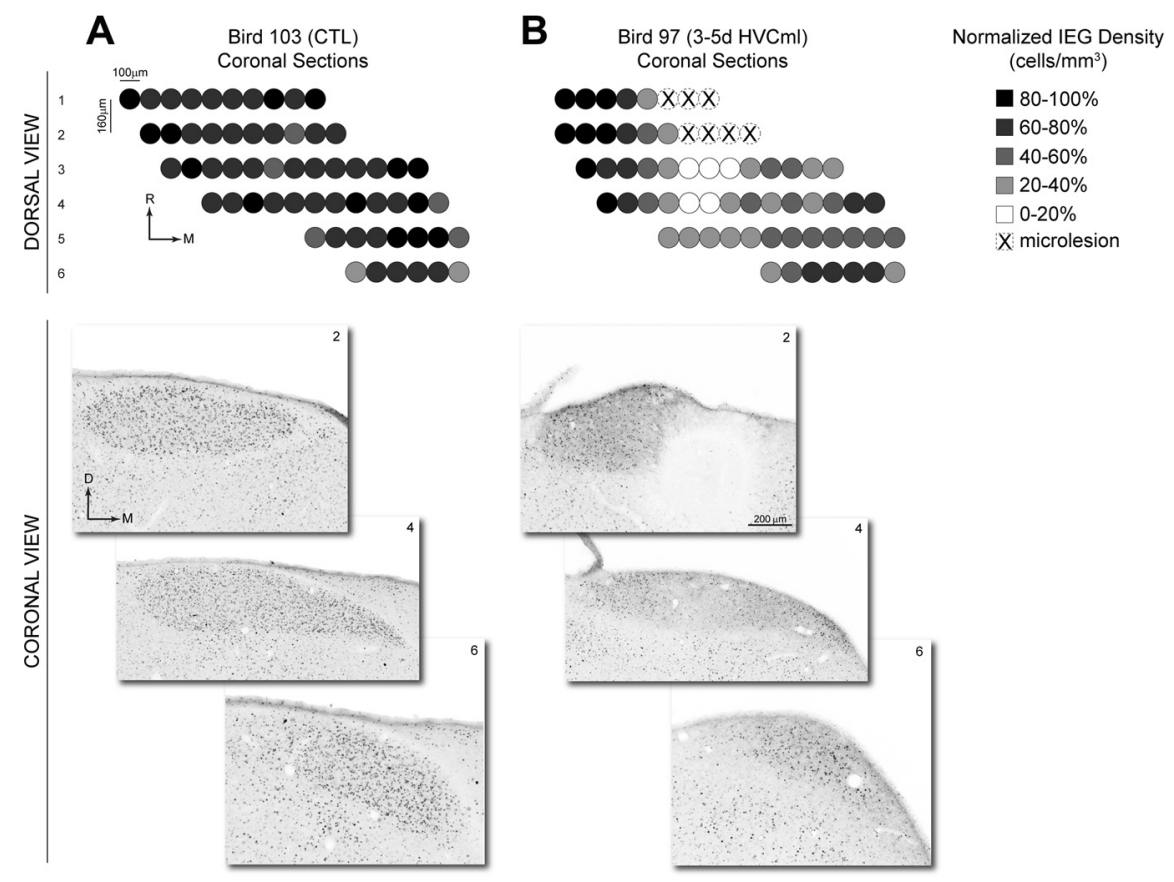

Figure 6. Quantification of the spatial organization of ZENK (IEG) labeling in coronal sections of HVC. The density of IEG labeling was measured in $100 \mu \mathrm{m}$ increments across the mediolateral axis of $n=6$ coronal tissue sections from a representative CTL bird $(\boldsymbol{A})$ and a representative $3-5 \mathrm{~d} \mathrm{HVCml}$ bird $(\boldsymbol{B})$. Individual tissue sections were $40 \mu \mathrm{m}$ thick and selected for analysis at $160 \mu \mathrm{m}$ density values were then rotated $90^{\circ}$ to provide a dorsal view of HVC (compare with Fig. 1). Bright-field images below show IEG labeling in sections 2, 4, and 6 from each bird, arranged according to their position relative to the midline. Under $B$, the bright-field image from section 2 of the $3-5 \mathrm{~d} \mathrm{HVCml}$ bird contains microlesion damage. $M$, Medial; $R$, rostral; D, dorsal.

ings and a second series of recordings was obtained. The pattern or results was unchanged after rotation.

\section{Results}

HVC microlesions

Analysis of the relationship between vocal recovery and IEG labeling requires that HVC microlesions be of similar size in all experimental groups. Table 1 presents a summary of the size of HVC microlesions and the percentage remaining HVC for each group (mean $\pm \mathrm{SE}$ ). No significant differences in bilateral microlesion volume were observed between groups (group: $F_{(1,12)}<1, p=$ $0.51)$. Comparison of HVC microlesion volume in left and right hemispheres within each group also revealed no significant differ- 


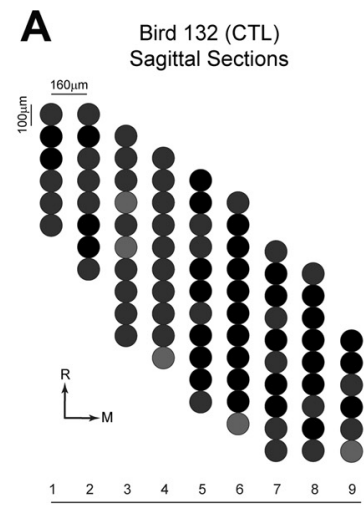

DORSAL VIEW

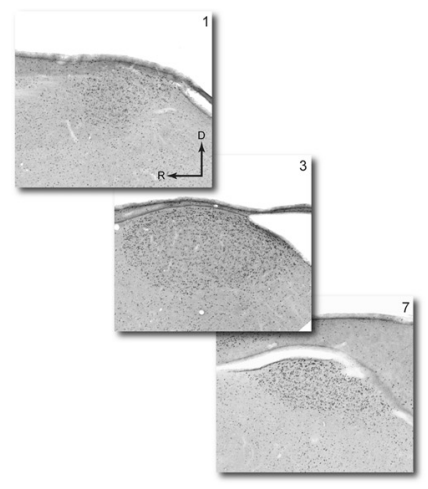

SAGITTAL VIEW
B

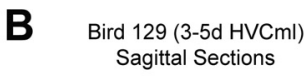
Sagittal Sections

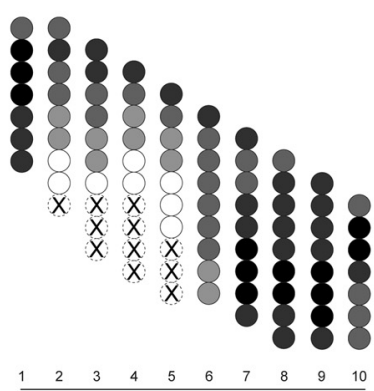

DORSAL VIEW

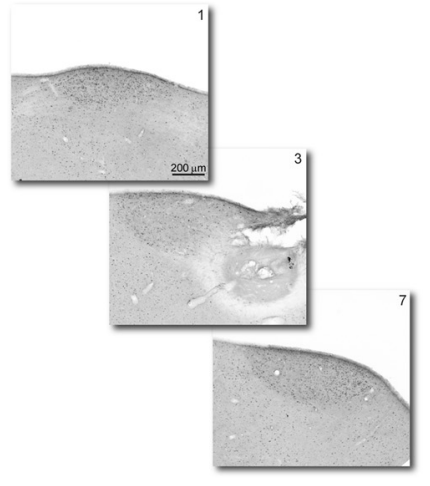

SAGITTAL VIEW

Figure 7. Quantification of the spatial organization of ZENK (IEG) labeling in sagittal sections of HVC. The density of IEG labeling was measured in $100 \mu \mathrm{m}$ increments across the rostrocaudal axis of sagittal tissue sections from a representative CTL bird $(\boldsymbol{A}, n=$ 9 tissue sections) and a representative $3-5 \mathrm{~d} \mathrm{HVCml}$ bird ( $\boldsymbol{B}, n=10$ tissue sections). Individual tissue sections were $40 \mu \mathrm{m}$ thick and selected for analysis at $160 \mu \mathrm{m}$ intervals. Density values were normalized to the $100 \mu \mathrm{m}$ increment with the highest labeling density in each bird. Normalized density values were then rotated $90^{\circ}$ to provide a dorsal view of HVC (compare with Fig. 1). Bright-field images below show IEG labeling in sections 1, 3, and 7 from each bird, arranged according to their position along the rostrocaudal axis. Under $\boldsymbol{B}$, the bright-field image from section 3 of the $3-5 \mathrm{~d} \mathrm{HVCml} \mathrm{bird} \mathrm{contains} \mathrm{microlesion} \mathrm{damage.} \mathrm{M,} \mathrm{Medial;}$ $R$, rostral; $D$, dorsal.

ences (3-5 d HVCml: $F_{(1,9)}=1.2, p=0.30 ; 2$ wk HVCml: $F_{(1,4)}<$ $1, p=0.74)$. These results indicate that the HVC microlesions were bilaterally symmetrical and that the relative size of the HVC microlesions did not differ between experimental groups.

\section{Vocal recovery after $\mathrm{HVC}$ microlesions}

Consistent with previous reports (Thompson and Johnson, 2007; Thompson et al., 2007), we found that HVC microlesions produce a transient loss of vocal patterning. Compared with singing produced at PRE1, both HVC microlesion groups showed a significant reduction in percentage similarity values at POST1 (Fig. 4). Percentage similarity values at FINAL for the 3-5d HVCml group remained significantly reduced compared with PRE1: these birds were still in varying stages of vocal recovery on their final day of singing. In contrast, percentage similarity values at FINAL for the $2 \mathrm{wk} \mathrm{HVCml}$ group showed a return to baseline (PRE1) levels, indicating recovery of the vocal pattern.

\section{Loss and recovery of IEG labeling in HVC after microlesions}

Figure 5 shows that the density of IEG labeling in HVC differed significantly between groups (group: $F_{(2,17)}=9.9, p=0.002$ ). This was entirely attributable to reduced IEG labeling in $3-5 \mathrm{~d}$ HVCml birds (3-5d HVCml vs 2 wk HVCml birds, $p=0.03$; $3-5 \mathrm{~d}$ HVCml vs CTL birds, $p=0.002$ ). IEG labeling in the $2 \mathrm{wk} \mathrm{HVCml}$ birds was not significantly different from CTL birds $(p=0.19)$.

These results indicate that HVC microlesions produce an initial reduction in the density of IEG labeling in HVC. However, after 2 weeks, the density of IEG labeling in HVC has returned to CTL levels.

\section{Rostrocaudal organization of IEG labeling in HVC}

Figure 5 shows that the density of IEG labeling in $\mathrm{HVC}$ was reduced by $>50 \%$ in the 3-5d HVCml group. Visual inspection of coronal or sagittal tissue sections from this group revealed that this reduction occurred in an asymmetrical manner. IEG labeling in individual tissue sections was visibly reduced across the thin dorsoventral axis of HVC within a region aligned to the mediolateral position of the microlesion. For example, Figure 3 shows a coronal section taken from the rostral half of an HVC in which the microlesion was located near the caudal pole of HVC (the dashed lines bracket the dorsoventral stripe of reduced labeling). The swath of reduced IEG labeling could be clearly observed in tissue sections throughout most of the rostrocaudal extent of $\mathrm{HVC}$ and occurred whether the microlesion was located near the caudal or rostral pole of HVC (i.e., the swath of reduced labeling was axial but not directional). Thus, the localized reduction in IEG labeling could not be solely attributable to transection of afferents that enter HVC rostrally. The greatest reduction in IEG labeling occurred in sections that were in close proximity to the microlesions, with the density of labeled cells gradually increasing with distance from the microlesion. In contrast, cell labeling medial and lateral to the microlesion was affected to a lesser degree, although there was a tendency in some birds for labeling lateral to the microlesion to be more robust.

Figure 6 shows quantification of spatial variation in the density of IEG labeling across the mediolateral and rostrocaudal axes of HVC from representative CTL $(A)$ and 3-5d HVCml $(B)$ birds. Tissue sections from these birds were collected in the coronal plane, and the density of IEG labeling was quantified in 100 $\mu \mathrm{m}$ increments across the $n=6$ tissue sections that contained HVC. The resulting normalized density values were then rotated $90^{\circ}$ for viewing from a dorsal perspective. The CTL $(A)$ shows an essentially uniform pattern of labeling density across mediolateral and rostrocaudal axes of HVC. In contrast, the 3-5d HVCml bird $(B)$ had microlesions positioned at the rostral pole of HVC and shows an axial decrease in IEG labeling that extends caudally from the position of the microlesion. IEG labeling medial to the position of the microlesion shows some reduction, whereas labeling lateral to the position of the microlesion is robust. Figure 7 shows quantification of IEG labeling across mediolateral and rostrocaudal axes of HVC from representative CTL $(A)$ and $3-5 \mathrm{~d}$ HVCml $(B)$ birds in which tissue sections were collected in the sagittal plane. The CTL $(A)$ again shows an essentially uniform pattern of IEG labeling density. The 3-5d HVCml bird ( $B)$, which had microlesions positioned at the caudal pole of HVC, shows an 

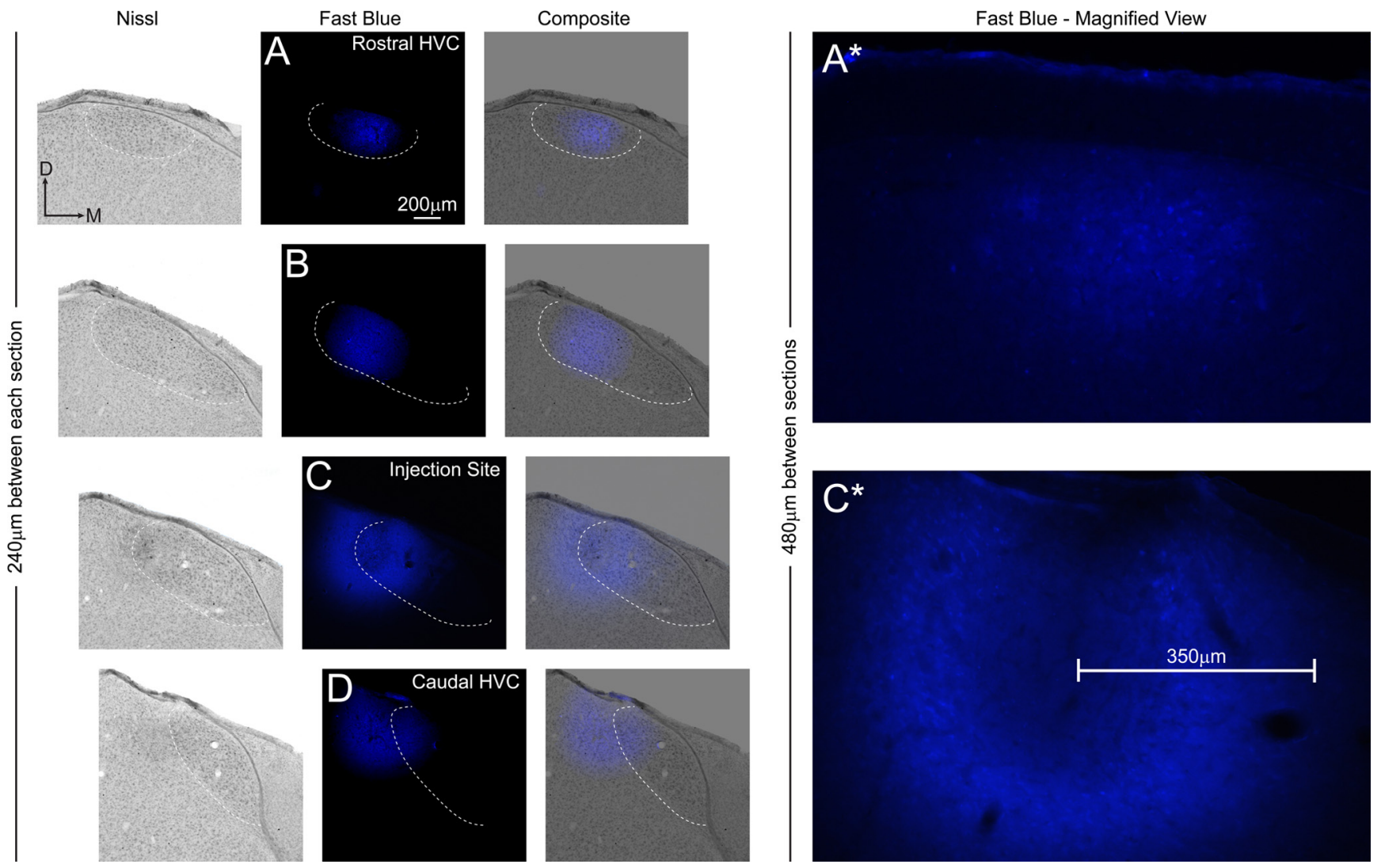

Figure 8. Rostrocaudal organization of HVC neural connectivity as demonstrated by focal injection of fast blue. At left, four coronal tissue sections $(\boldsymbol{A}-\boldsymbol{D})$ selected at $240 \mu \mathrm{m}$ increments span the rostrocaudal axis of HVC. Each section is shown under bright-field (Nissl) and fluorescent (fast blue) illumination. The third column (Composite) shows composites of bright-field and fluorescent images from each section. In all images, the Nissl-defined borders of HVC are indicated by a white dashed line. The horizontal offset in each successive row of images accommodates the medial shift that occurs between rostral and caudal poles of HVC (see Fig. 1). In this bird, a laterally situated dye injection near caudal HVC ( $($ ) produces retrograde labeling within the medial half of rostral HVC (A), indicating a pattern of retrograde labeling aligned to the rostrocaudal axis of HVC. The right panel (Fast Blue - Magnified View) shows high-magnification images of fast blue labeling in sections that were adjacent (in the rostral direction) to $A$ and $C$ in the left. The scale bar in $C$ * shows that the radius of passive diffusion of fast blue away from the injection site was $\sim 350 \mu \mathrm{m}$. The retrograde cell labeling in rostral HVC $\left(\boldsymbol{A}^{*}\right)$ is located at a distance of $480 \mu \mathrm{m}$ away from the injection site, exceeding the radius of passive diffusion of the dye. Overexposure of the fast blue injection sites in $\boldsymbol{C}$ and $C^{*}$ ensured capture of cell labeling medial to the injection site; such labeling was limited in extent compared with the labeling present in rostral HVC. D, Dorsal; M, medial.

axial decrease in IEG labeling that extends rostrally from the position of the microlesion. Labeling medial and lateral to the position of the microlesion were affected to a lesser degree.

Because the expression of IEGs depends on neural activity, one possibility is that microlesions produce a selective deafferentation of HVC neurons located rostral and/or caudal to the mediolateral position of the microlesion, resulting in a reduction in the excitatory drive necessary to reach the threshold for IEG expression. Selective rostrocaudal deafferentation would also explain why IEG labeling lateral or medial to the microlesion site was affected to a lesser degree. Thus, the spatial pattern of IEG labeling produced by microlesions suggests that HVC contains an asymmetric neural network characterized by greater levels of rostrocaudal than mediolateral connectivity. Importantly, this axial organization does not appear directional because microlesions at rostral (Fig. 6) or caudal (Fig. 7) poles of HVC produced swaths of reduced IEG labeling that extended caudally or rostrally, respectively. It seems therefore unlikely that this organization is imposed solely by afferent input that enters along the rostral edge of HVC.

\section{Rostrocaudal organization of HVC neural connectivity}

The spatial pattern of IEG labeling in the $3-5 \mathrm{~d} H V C m l$ group could be explained if HVC is anatomically organized such that the intranuclear axons of individual HVC neurons show greater extension and elaboration within the rostrocaudal axis. This possibility was investigated with focal infusions of retrograde tracttracing dyes into HVC. If the IEG findings are indicative of underlying HVC anatomy, then such infusions should produce a pattern of retrograde labeling that extends farther within the rostrocaudal than the mediolateral axis of HVC.

Figures 8 and 9 show typical results obtained from focal infusions of the retrograde tract-tracing dyes fast blue (Fig. 8) and $\mathrm{DiO}$ (Fig. 9) into HVC. Retrograde cell labeling extended rostrally and/or caudally from the focal dye infusion with limited transport of dye across the mediolateral axis. Similar to the IEG results in the 3-5d HVCml group, retrograde labeling formed a rostrocaudal swath aligned with the mediolateral position of the focal dye infusion. The same pattern was observed with all tracers used, with injection sites located within rostrocaudal axis of central HVC. Therefore, as suggested by the IEG findings, the axons of HVC neurons appear to extend and elaborate primarily within the rostrocaudal axis, with more limited extension into the mediolateral axis.

Retrograde labeling within the nucleus interface (Nlf, a telencephalic nucleus that provides afferent input to HVC and that is located ventral and rostral to HVC at a distance of $\sim 1.5 \mathrm{~mm}$ ) served as a positive control, demonstrating that sufficient time had elapsed for complete retrograde filling of neurons within HVC. Labeling was also observed in paraHVC, a region adjacent to HVC (located along the dorsomedial edge of the nidopallium) 


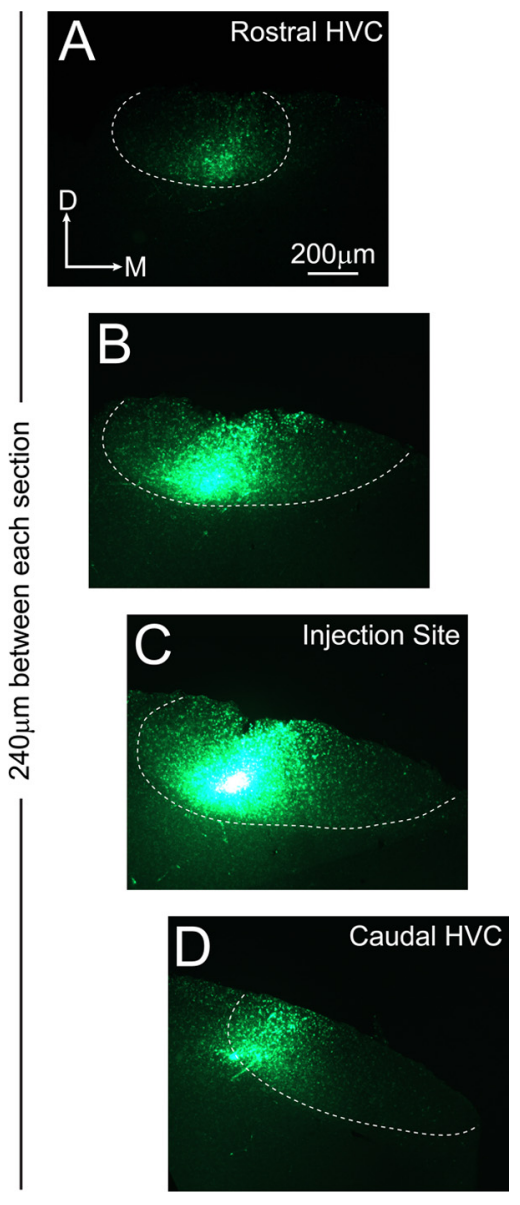

Figure 9. Rostrocaudal organization of HVC neural connectivity as demonstrated by focal injection of Di0. Four coronal tissue sections $(\boldsymbol{A}-\boldsymbol{D})$ selected at $240 \mu \mathrm{m}$ increments span the rostrocaudal axis of HVC and are shown under fluorescent illumination. The Niss--defined borders of HVC (obtained after counterstaining of each section) are indicated by a white dashed line. The horizontal offset in the images accommodates the medial shift that occurs between rostral and caudal poles of HVC (see Fig. 1). In this bird, a centrally situated dye injection near caudal HVC (C) produces retrograde labeling within the lateral half of caudal HVC (D) and within the medial half of rostral HVC $(\boldsymbol{A})$, indicating a pattern of retrograde labeling aligned to the rostrocaudal axis of HVC. Note that Di0 is a hydrophobic tracer and it therefore produced more focal injection sites compared with fast blue (see Fig. 8C). Overexposure of the Di0 injection site $(C)$ ensured capture of labeling lateral and medial to the injection site; such labeling was limited in extent compared with the labeling present in caudal and rostral HVC. D, Dorsal; M, medial.

that also sends afferent input to HVC (Foster and Bottjer, 1998). The observation that infusion of several different retrograde dyes all produce labeling that extends primarily within the rostrocaudal axis of HVC finds support in the previous observations of Nottebohm et al. (1982), in which HRP injections indicated that HVC neurons are connected with one another primarily within the rostrocaudal axis.

\section{Rostrocaudal organization of HVC electrophysiology}

Both IEG and tract-tracing data suggest a rostrocaudal organization of HVC anatomy. To test whether this organization is also reflected in HVC electrophysiology, horizontal slices of HVC were obtained, and local field potentials were recorded in various directions relative to the placement of a bipolar stimulating electrode. An example of the placement of the stimulating and recording electrodes is displayed schematically in Figure $10 A^{*}$. Robust local field potentials were obtained when the recording electrode was placed in the rostrocaudal axis (Fig. 10B, R2). When the stimulation was held constant and the same recording electrode was repositioned an equal distance away in the mediolateral axis relative to the stimulating electrode, there was little, if any, driven population activity (Fig. $10 \mathrm{~B}, \mathrm{R} 5)$. Average $(n=20$ traces) peak-to-peak amplitudes for both presynaptic and postsynaptic components of the traces are also shown in Figure 10C. In this example, robust responses were recorded both rostral and caudal to the stimulating electrode but not medial to the stimulation. Figure 11 displays the magnitude of the presynaptic $(A)$ and postsynaptic $(B)$ responses as a function of angle from the stimulating electrode for each individual slice, in which $0^{\circ}$ is either the medial or lateral direction. As can be seen, the rostrocaudal bias was consistently observed. In one case, the slice was rotated $180^{\circ}$ after the first series of recordings, and the rostrocaudal bias was clear relative to both the medial and lateral directions. There was no apparent directionality of the rostrocaudal bias; some slices showed larger responses rostrally than caudally, and others showed the opposite trend. In all cases, however, the largest responses were seen in the rostrocaudal axis compared with the mediolateral axis. For statistical analysis, the amplitudes of the averaged field potentials at the equidistant medial/lateralmost and rostral/caudal-most locations were compared using paired $t$ tests. If two (e.g., a rostral-most and caudal-most) locations were recorded for a given slice, the responses at those two locations were averaged for statistical comparisons. Mean peakto-peak amplitudes of the presynaptic and postsynaptic responses are shown in Figure $11 C$. There was a statistically reliable difference in the size of both the presynaptic field potentials $\left(t_{(4)}=\right.$ $6.5, p<0.01)$ and the postsynaptic field potentials $\left(t_{(4)}=5.4, p<\right.$ 0.0 ). Although some connectivity appears to be present in the mediolateral axis, the lack of robust field potentials suggests that this mediolateral connectivity is relatively sparse. Together, these data suggest that the bulk of electrophysiological connectivity within $\mathrm{HVC}$ is oriented in the rostrocaudal axis.

\section{Discussion}

Our goal in these experiments was to elucidate the network architecture of HVC, a pallial region analogous to cortex in mammals that encodes the learned sequential vocal pattern in songbirds. To do this, we combined focal ablation with IEG labeling to visualize singing-related neural activity within HVC. Disruption of HVC network activity by focal ablation revealed an asymmetrically organized architecture. That is, despite the much greater mediolateral extent of HVC anatomy (Fig. 1), we observed a patterned reduction in IEG labeling that extended farther within the rostrocaudal axis than the mediolateral axis. The IEG findings could be explained if synaptic connectivity extends and elaborates primarily within the rostrocaudal axis of HVC. Tract-tracing and in vitro electrophysiological experiments confirmed the rostrocaudal organization suggested by the IEG experiment. Thus, findings derived from three different techniques indicate that the network architecture of HVC is organized by anatomy and physiology to convey the bulk of neural signaling within the rostrocaudal axis. This axial organization did not appear to be directional. Vectors of reduced IEG labeling and neural connectivity appeared symmetrical in both rostral and caudal directions, and electrophysiological responses showed no consistent rostral versus caudal bias. The limited spatial extent of mediolateral connectivity was a consistent result across all three techniques. Together, our findings suggest a specific axial orientation to HVC network activity during singing. 


\section{Relationship to HVC physiology during singing}

The role of HVC in encoding the temporal organization of the zebra finch vocal pattern has been well established by Fee and colleagues (Hahnloser et al., 2002; Long and Fee 2008; Long et al., 2010). Recent evidence suggests that the sequential ordering of vocal sounds within and across song syllables is driven by a sequential ordering of HVC neural activity (Long et al., 2010). However, this sequential neural activity need not be directionally linear within HVC or even confined to a particular anatomical axis of HVC. Indeed, neuronal cell bodies within the large central region of HVC show little if any indication of topographic organization, appearing instead to be a mosaic of cells with evidence of local clustering (Fortune and Margoliash, 1995; Wild et al., 2005). Moreover, a mapping of the IEG response after singing reveals widespread activation throughout the entirety of the HVC (Jarvis and Nottebohm, 1997; Jin and Clayton, 1997; Jarvis et al., 1998; replicated here in Figs. 6A, 7A).

Thus, our finding of an axial organization in HVC population activity (evidenced by IEG as well as electrophysiological data) is the first to suggest that the population synaptic architecture of HVC forms an axially organized spatial substrate for the sequential ordering of neural activity during the motor act of singing. Whether a similar axial organization might be observed within cortical regions that control vocal sequences in humans (Bohland et al., 2010; Price, 2010) is an intriguing question for future research. Although our findings do not specify how the sequence of syllables in the vocal pattern might be mapped to this spatial substrate, it seems unlikely that syllables are sequentially arranged across the rostrocaudal axis HVC (Hahnloser et al., 2002). Instead, this spatial substrate could be used to organize axonal delays that would synchronize activity across an axially organized population of HVC neurons to control the timing and/or amplitude of vocal production. The short-range mediolateral connectivity of HVC might serve to synchronize or link axonal delays oriented within the rostrocaudal axis of adjacent regions of HVC.

Axonal delays are known to be involved in the coding of acoustic sensory information by promoting synchronous activation of specific neurons (Carr and Konishi, 1988; Overholt et al., 1992; Joseph and Hyson, 1993). Thus, axonal delays intrinsic to HVC might promote the synchronous presynaptic excitation that appears necessary to elicit the sparse, precisely timed bursting of RA-projecting HVC neurons during singing (Long et al., 2010) or the rhythmic activity that can be driven in HVC in vitro

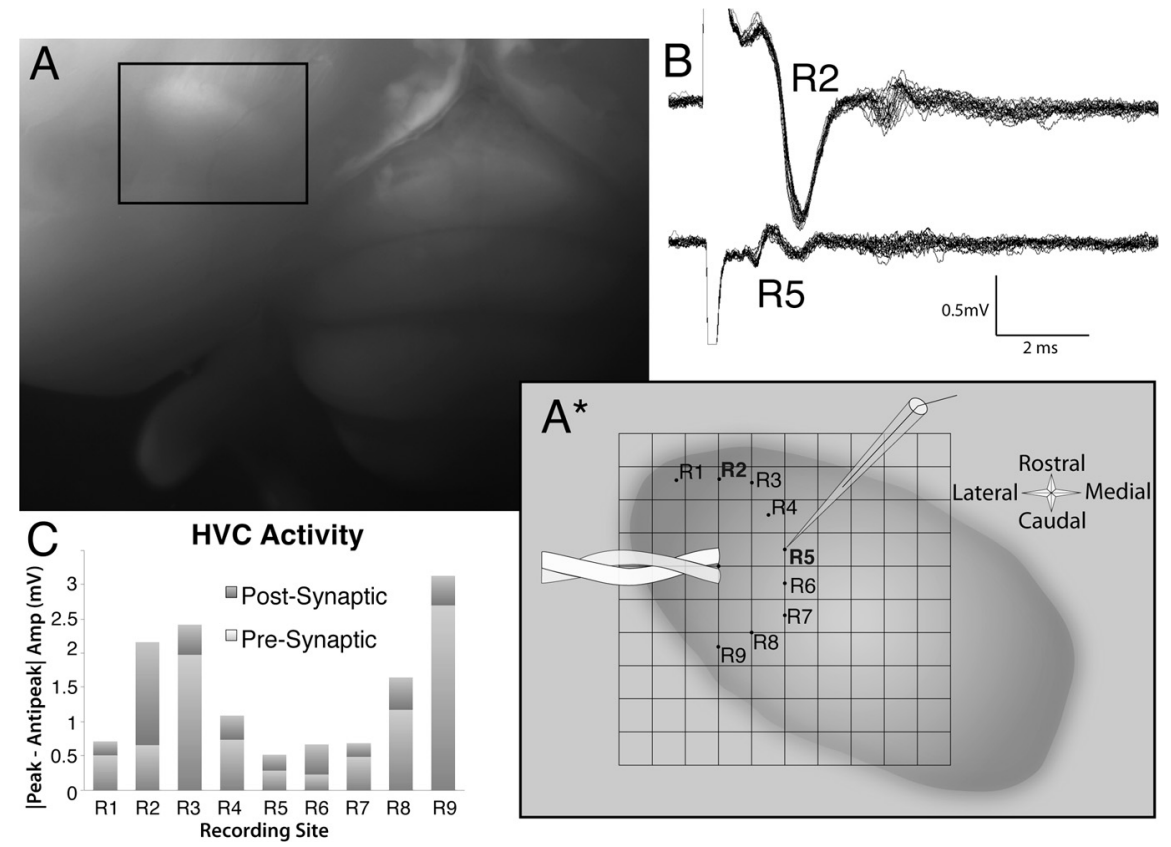

Figure 10. Example of the in vitro stimulation and recording experiments in horizontal slices of HVC suggesting that the bulk of electrophysiological connectivity within HVC is oriented in the rostrocaudal axis. $A$, After resection of the hippocampus, HVC is readily identified by a distinct bulge on the dorsal surface of the telencephalon (compare with Fig. 1 ). $A^{*}$, Schematic of HVC drawn from the whole-brain image in $\boldsymbol{A}$ includes an overlay depicting the placement of the stimulating and recording electrodes in horizontal slices of HVC. Recordings were made in an arc around the stimulating electrode, allowing comparisons of response amplitudes in the same slice. $\boldsymbol{B}$, Sample recordings demonstrating the robust field potentials observed in the rostrocaudal axis (R2) compared with those observed in the mediolateral axis (R5) using the same stimulus conditions. Twenty sweeps are overlaid, demonstrating the consistency of the responses. C, Quantification of the peak-to-peak amplitudes of the both presynaptic and postsynaptic components of the recordings confirmed this rostrocaudal bias.

A

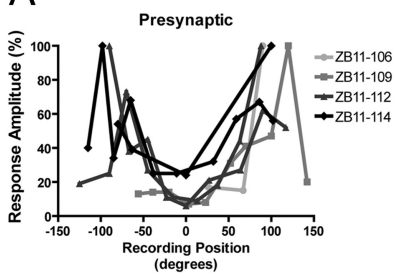

Figure 11. Normalized peak-to-peak amplitudes of the presynaptic $(\boldsymbol{A})$ and postsynaptic $(\boldsymbol{B})$ components of the average field potentials for individual slices as a function of angle from the site of stimulation $(n=4)$. Recordings from each slice were normalized as a proportion of the size of the largest response. Zero degrees represents recordings from locations either directly medial or directly lateral to the stimulating electrode; hence, $90^{\circ}$ represents a location that is directly caudal to the stimulation site, and $-90^{\circ}$ represents a location directly rostral to the stimulation site. In every slice, there was an obvious rostrocaudal bias in the amplitude of the fields, but there was no consistent preference for rostral direction compared with the caudal direction across slices. One slice (ZB11-112) was rotated $180^{\circ}$, and a second series of recordings was performed. Similar patterns of results were obtained in both conditions, showing that there is rostrocaudal bias when compared with either the medial or lateral locations of the slice. Another slice (ZB11-114) had a second series of recordings performed at a greater distance from the stimulating electrode, and the rostrocaudal bias was observed at both distances. C, Mean \pm SEM peak-to-peak amplitudes of the presynaptic and postsynaptic components of the rostrocaudal-most and mediolateral-most recording locations $(n=5)$. The size of the field potential for both components was reliably greater (paired $t$ test, $p<0.05$ ) at the rostrocaudal recording locations. ML, Mediolateral; RC, rostrocaudal.
(Solis and Perkel, 2005). Anything that disrupts this timingmicrostimulation ( $\mathrm{Vu}$ et al., 1994), microlesions (Thompson and Johnson, 2007), local cooling (Long and Fee, 2008)would then disrupt the ability of HVC to synchronize activity across the population and lead to altered patterning. If such timing processes exist in HVC, our data suggest that connectivity in the rostrocaudal axis would be the primary contributor, and disruption of information flow in this axis would be 
more detrimental than disruption of information flow in the mediolateral axis.

\section{Relationship between IEG recovery and vocal recovery}

Zebra finch vocal patterns show surprising resilience after HVC microlesions, typically recovering to preoperative structure in $\sim 1$ week (Thompson and Johnson, 2007; Thompson et al., 2007). In the present study, recovery of IEG labeling within HVC was observed in the $2 \mathrm{wk} \mathrm{HVCml}$ birds (Fig. 5), particularly within the rostrocaudal swath of reduced labeling observed in the $3-5 \mathrm{~d}$ HVCml group. Thus, HVC network activity (as indexed by IEG labeling) also appears to be quite resilient after focal damage, although we emphasize that this applies only to the spatial distribution of IEG labeling within HVC (i.e., IEG data are insufficient to tell whether a normal pattern of neural activity also recovered). The relatively rapid rate at which IEG labeling is restored within HVC could potentially involve several mechanisms, including neuronal addition (adult neurogenesis is well established in zebra finches; Nottebohm, 2004), homeostatic synaptic plasticity (Pozo and Goda, 2010), and/or lesion-induced axonal sprouting from neighboring regions (Deller et al., 2006).

The correlation between IEG recovery and vocal recovery suggests that the former might underlie the latter. However, recovery of IEG labeling within HVC can be disassociated from vocal recovery. When HVC microlesions are combined with deafening, birds cannot recover their preoperative vocal patterns (Thompson et al., 2007). In a separate group of birds ( $n=5$; data not shown), we combined HVC microlesions with deafening followed by a 2 week recovery period. As expected, these birds did not recover their vocal patterns. However, when processed for IEG immunohistochemistry, we found that the density of IEG labeling within HVC was not different from CTL birds or $2 \mathrm{wk}$ HVCml birds. Thus, although the recovery of IEG labeling within HVC may be necessary for vocal recovery after microlesions, it is not sufficient for vocal recovery. Other studies (Thompson and Johnson, 2007; Thompson et al., 2007) suggest that vocal recovery from HVC microlesions involves modulation of activity within the anterior premotor pathway, which was not examined in this study.

\section{Limitations of the study}

In all experiments, we focused on the overall population architecture of HVC. However, it is well established that HVC contains at least three hodologically defined subpopulations of neurons (RA-projecting, Area X-projecting interneurons) arranged in a superficially isomorphic mosaic (Wild et al., 2005). Moreover, three cytoarchitectonically distinct subregions of HVC have been described-a large central region flanked by smaller zones occupying the ventral-caudomedial and dorsolateral edges of Nissl-defined HVC (Fortune and Margoliash, 1995). All three subregions contain a mosaic of interneurons and RA- and Area $\mathrm{X}$-projecting neurons, but characteristic differences in cell size, shape, and orientation delineate each subregion. With respect to the placement of microlesions, dye injections, and electrophysiological stimulation and recording, our findings address the large central region of $\mathrm{HVC}$, and it is possible that the organization of the smaller ventral-caudomedial and dorsolateral zones could differ from the rostrocaudal organization described here. Similarly, our findings do not specify whether the three hodologically defined subpopulations of HVC neurons differed or were similar in their contribution to our IEG, tract-tracing, and electrophysiological results. Although detailed information on the extent of intranuclear axon arbors for each HVC subpopulation is some- what limited, Mooney (2000) described the anatomy of a small sample of neurons from each subpopulation, and Benton et al. (1998) described the anatomy of Area X-projecting neurons. The available data suggest that the axon arbors of HVC interneurons are quite local, whereas the collateral (intranuclear) axons of RAand Area X-projecting neurons elaborate throughout the dorsoventral and rostrocaudal axes of HVC (the mediolateral axis was not examined). Thus, in the specific case of our tract-tracing experiment, cells filled at the most distal locations from the injection sites were likely from one or both subpopulations of HVC projection neurons. Overall, the present findings highlight the need to reexamine the anatomy of individual HVC cell types in greater detail, particularly to establish the composition of the cell populations that contribute to the rostrocaudal organization of HVC.

\section{References}

Andalman AS, Fee MS (2009) A basal ganglia-forebrain circuit in the songbird biases motor output to avoid vocal errors. Proc Natl Acad Sci U S A 106:12518-12523.

Aronov D, Andalman AS, Fee MS (2008) A specialized forebrain circuit for vocal babbling in the juvenile songbird. Science 320:630-634.

Benton S, Cardin JA, DeVoogd TJ (1998) Lucifer Yellow filling of area $\mathrm{X}$-projecting neurons in the high vocal center of female canaries. Brain Res 799:138-147.

Bohland JW, Bullock D, Guenther FH (2010) Neural representations and mechanisms for the performance of simple speech sequences. J Cogn Neurosci 22:1504-1529.

Brainard MS, Doupe AJ (2000) Interruption of a basal ganglia-forebrain circuit prevents plasticity of learned vocalizations. Nature 404:762-766.

Carr CE, Konishi M (1988) Axonal delay lines for time measurement in the owl's brainstem. Proc Natl Acad Sci U S A 85:8311-8315.

Deller T, Haas CA, Freiman TM, Phinney A, Jucker M, Frotscher M (2006) Lesion-induced axonal sprouting in the central nervous system. Adv Exp Med Biol 557:101-121.

Fortune ES, Margoliash D (1995) Parallel pathways and convergence onto $\mathrm{HVc}$ and adjacent neostriatum of adult zebra finches (Taeniopygia guttata). J Comp Neurol 360:413-441.

Foster EF, Bottjer SW (1998) Axonal connections of the high vocal center and surrounding cortical regions in juvenile and adult male zebra finches. J Comp Neurol 397:118-138.

Hahnloser RH, Kozhevnikov AA, Fee MS (2002) An ultra-sparse code underlies the generation of neural sequences in a songbird. Nature 419:65-70.

Jarvis ED, Nottebohm F (1997) Motor-driven gene expression. Proc Natl Acad Sci U S A 94:4097-4102.

Jarvis ED, Scharff C, Grossman MR, Ramos JA, Nottebohm F (1998) For whom the bird sings: context-dependent gene expression. Neuron 21:775-788.

Jin H, Clayton DF (1997) Localized changes in immediate-early gene regulation during sensory and motor learning in zebra finches. Neuron 19:1049-1059.

Joseph AW, Hyson RL (1993) Coincidence detection by binaural neurons in the chick brain stem. J Neurophysiol 69:1197-1211.

Long MA, Fee MS (2008) Using temperature to analyze temporal dynamics in the songbird motor pathway. Nature 456:189-194.

Long MA, Jin DZ, Fee MS (2010) Support for a synaptic chain model of neuronal sequence generation. Nature 468:394-399.

Margoliash D (1986) Preference for autogenous song by auditory neurons in a song system nucleus of the white-crowned sparrow. J Neurosci 6:1643-1661.

Mello CV, Ribeiro S (1998) ZENK protein regulation by song in the brain of songbirds. J Comp Neurol 393:426-438.

Mooney R (2000) Different subthreshold mechanisms underlie song selectivity in identified $\mathrm{HVc}$ neurons of the zebra finch. J Neurosci 20:5420-5436.

Mooney R, Konishi M (1991) Two distinct inputs to an avian song nucleus activate different glutamate receptor subtypes on individual neurons. Proc Natl Acad Sci U S A 88:4075-4079.

Nottebohm F (2004) The road we travelled: discovery, choreography, and 
significance of brain replaceable neurons. Ann N Y Acad Sci 1016: 628-658.

Nottebohm F, Kelley DB, Paton JA (1982) Connections of vocal control nuclei in the canary telencephalon. J Comp Neurol 207:344-357.

Olson CR, Rodrigues PV, Jeong JK, Prahl DJ, Mello CV (2011) Organization and development of zebra finch HVC and paraHVC based on expression of zRalDH, an enzyme associated with retinoic acid production. J Comp Neurol 519:148-161.

Overholt EM, Rubel EW, Hyson RL (1992) A circuit for coding interaural time differences in the chick brainstem. J Neurosci 12:1698-1708.

Pozo K, Goda Y (2010) Unraveling mechanisms of homeostatic synaptic plasticity. Neuron 66:337-351.

Price CJ (2010) The anatomy of language: a review of 100 fMRI studies published in 2009. Ann N Y Acad Sci 1191:62-88.

Solis MM, Perkel DJ (2005) Rhythmic activity in a forebrain vocal control nucleus in vitro. J Neurosci 25:2811-2822.

Sutter ML, Margoliash D (1994) Global synchronous response to autogenous song in zebra finch HVc. J. Neurophysiol 72:2105-2123.

Tchernichovski O, Nottebohm F, Ho CE, Pesaran B, Mitra PP (2000) A procedure for an automated measurement of song similarity. Anim Behav 59:1167-1176.

Thompson JA, Johnson F (2007) HVC microlesions do not destabilize the vocal patterns of adult male zebra finches with prior ablation of LMAN. Dev Neurobiol 67:205-218.

Thompson JA, Wu W, Bertram R, Johnson F (2007) Auditory-dependent vocal recovery in adult male zebra finches is facilitated by lesion of a forebrain pathway that includes the basal ganglia. J Neurosci 27: $12308-12320$.

Vu ET, Mazurek ME, Kuo YC (1994) Identification of a forebrain motor programming network for the learned song of zebra finches. J Neurosci 14:6924-6934.

Whitney O, Johnson F (2005) Motor-induced transcription but sensoryregulated translation of ZENK in socially interactive songbirds. J Neurobiol 65:251-259.

Whitney O, Soderstrom K, Johnson F (2000) Post-transcriptional regulation of zenk expression associated with zebra finch vocal development. Brain Res Mol Brain Res 80:279-290.

Wild JM, Williams MN, Howie GJ, Mooney R (2005) Calcium-binding proteins define interneurons in HVC of the zebra finch (Taeniopygia guttata). J Comp Neurol 483:76-90.

Wu W, Thompson JA, Bertram R, Johnson F (2008) A statistical method for quantifying songbird phonology and syntax. J Neurosci Methods 174: $147-154$. 